\title{
Transient rotating flow over a moving surface with a magnetic field
}

\author{
M. Kumari *, G. Nath \\ Department of Mathematics, Indian Institute of Science, Bangalore 560012, India
}

\begin{abstract}
The transient flow and heat transfer on a moving surface in a rotating fluid in the presence of a magnetic field have been investigated. The unsteadiness in the flow field has been introduced by the sudden change in the surface velocity or the fluid angular velocity. The parabolic partial differential equations governing the unsteady flow and heat transfer have been solved by using an implicit finite-difference scheme in combination with the quasilinearization technique. The computations have been carried out from the initial steady state to the final steady state. The effects of the sudden change in the surface velocity on the flow and heat transfer are found to be more significant than those of the impulsive change in the angular velocity of the fluid. When the surface velocity is suddenly reduced, the surface shear stress is found to vanish in a small time interval after the start of the impulsive motion, but it does not imply flow separation. The surface shear stress for the primary flow increases with the magnetic field and the fluid angular velocity, but the surface heat transfer decreases. The surface shear stress for the secondary flow increases with the angular velocity of the fluid, but decreases with increasing magnetic field.
\end{abstract}

\section{Introduction}

The flow and heat transfer problem due to a moving surface in an ambient fluid is important in many industrial processes such as the cooling of a hot metallic plate in a cooling bath, the extrusion of a plastic sheet, glass blowing, continuous casting and spinning of fibers. Sakiadis [1] was the first to study the flow induced by a surface moving with a constant velocity in an ambient fluid. The corresponding heat transfer problem was considered by Tsou et al. [2]. Crane [3] studied the same prob-

\footnotetext{
* Corresponding author. Tel.: +91 802293 3214/2265; fax: +918023600146.

E-mail address: mkumari@math.iisc.ernet.in (M. Kumari).
}

lem as in [1], but assumed that the surface velocity varies linearly with the streamwise distance $x\left(U_{0}=a x, a>0\right)$. Subsequently, Carragher and Crane [4] investigated the corresponding heat transfer problem.

In recent years, problems involving magnetic field have become important. Many metallurgical processes such as drawing, annealing and tinning of copper wire involve the cooling of continuous strips or filaments by drawing them through an ambient fluid. By drawing these filaments in an electrically conducting fluid under the influence of an applied magnetic field, the rate of cooling can be controlled. The flow and (or) heat transfer over a moving surface in the presence of a magnetic field have been investigated by Chakrabarti and Gupta [5], Andersson [6], Vajravelu and Hadjinicolaou [7], Pop and $\mathrm{Na}$ [8] and Kumari and Nath [9]. Wang [10] 


\begin{tabular}{|c|c|c|c|}
\hline \multicolumn{4}{|c|}{ Nomenclature } \\
\hline $\begin{array}{l}a \\
B\end{array}$ & $\begin{array}{l}\text { velocity gradient, } \mathrm{s}^{-1} \\
\text { magnetic field, } \mathrm{kg}^{1 / 2} \mathrm{~m}^{-1 / 2} \mathrm{~s}^{-1}\end{array}$ & $x, y, z$ & Cartesian coordinates, $\mathrm{m}$ \\
\hline$C_{\mathrm{f} x}$ & local skin friction coefficient for the primary & \multicolumn{2}{|c|}{ Greek symbols } \\
\hline & flow & $\alpha$ & thermal diffusivity of the fluid, $\mathrm{m}^{2} \mathrm{~s}^{-1}$ \\
\hline$C_{\mathrm{f} z}$ & $\begin{array}{l}\text { local skin friction coefficient for the second- } \\
\text { ary flow }\end{array}$ & $\begin{array}{l}\epsilon_{1}, \epsilon_{2} \\
\eta\end{array}$ & $\begin{array}{l}\text { dimensionless constants } \\
\text { dimensionless transformed coordinate }\end{array}$ \\
\hline$f$ & dimensionless stream function & $\theta$ & dimensionless temperature \\
\hline$f^{\prime}$ & dimensionless velocity for the primary flow & $\lambda$ & dimensionless angular velocity of the fluid \\
\hline$L$ & characteristic length, $\mathrm{m}$ & $\mu$ & coefficient of fluid viscosity, $\mathrm{kg} \mathrm{m}^{-1} \mathrm{~s}^{-1}$ \\
\hline$M$ & dimensionless magnetic parameter & $\mu_{0}$ & magnetic permeability \\
\hline$N u_{x}$ & local Nusselt number & $v$ & kinematic fluid viscosity, $\mathrm{m}^{2} \mathrm{~s}^{-1}$ \\
\hline $\operatorname{Pr}$ & Prandtl number & $\rho$ & density of the fluid, $\mathrm{kg} \mathrm{m}^{-3}$ \\
\hline$R e_{x}$ & local Reynolds number & $\sigma$ & electrical conductivity, $\mathrm{m}^{-2} \mathrm{~s}$ \\
\hline$R e_{\mathrm{m}}$ & magnetic Reynolds number & $\Omega$ & angular velocity of the fluid at $t^{*}>0, \mathrm{~m} \mathrm{~s}^{-1}$ \\
\hline$s$ & $\begin{array}{l}\text { dimensionless velocity for the secondary } \\
\text { flow }\end{array}$ & $\Omega_{0}$ & angular velocity of the fluid at $t^{*}=0, \mathrm{~ms}^{-1}$ \\
\hline$t$ & time, $s$ & \multicolumn{2}{|c|}{ Subscripts } \\
\hline$t^{t \prime}$ & $\begin{array}{l}\text { dimensionless time } \\
\text { temperature, } \mathrm{K}\end{array}$ & $i, w, \infty$ & initial conditions, conditions at the wall and \\
\hline$T_{\mathrm{w}}$ & wall temperature, $\mathrm{K}$ & $t, x=$ & $\begin{array}{l}\text { ambient conditions, respectively } \\
\text { derivatives with respect to } t, x \text { and } z \text {, respec- }\end{array}$ \\
\hline$T_{\infty}$ & ambient temperature, $\mathrm{K}$ & $i, x, 2$ & $\begin{array}{l}\text { tively } \\
\text { tives we }\end{array}$ \\
\hline$u, v, w$ & $\begin{array}{l}\text { velocity components along } x, y \text { and } z \text { direc- } \\
\text { tions, respectively, } \mathrm{ms}^{-1}\end{array}$ & & \\
\hline$U$ & surface velocity at $t>0, \mathrm{~m} \mathrm{~s}^{-1}$ & \multicolumn{2}{|c|}{ Superscript } \\
\hline$U_{0}$ & surface velocity at $t=0, \mathrm{~ms}^{-1}$ & & prime denotes derivative with respect to $\eta$ \\
\hline
\end{tabular}

has studied the flow over a moving surface in a rotating fluid. These studies deal with steady flows. However, in many situations, the flow may be unsteady due to the sudden change in the surface velocity or fluid angular velocity. Takhar and Nath [11] have obtained self-similar solutions of the unsteady flow and heat transfer over a stretching surface in the presence of a magnetic field where the surface velocity and the angular velocity of the fluid vary inversely as a linear function of time.

The boundary layer flow and heat transfer problem over a moving surface differs from that over a stationary surface caused by the free stream velocity due to the entrainment of the fluid. The moving surface prevents or delays the separation of the boundary layer from the wall by injecting momentum in the existing boundary layer. The surface shear stress and the heat transfer for the moving surface are found to be about $50 \%$ higher than those of the classical boundary layer flow over a flat plate. The magnetic field and the rotation of the fluid increase the surface shear stress for the primary flow, but reduce the surface heat transfer. On the other hand, the magnetic field and the rotation of the fluid exert opposite effect on the surface shear stress for the secondary flow. Hence, the unsteady flow over a moving surface in a rotating fluid in the presence of a magnetic field is an interesting problem.
The aim of this analysis is to study the transient flow and heat transfer characteristics over a moving surface in a rotating fluid in the presence of a magnetic field. We have considered the situation where there is an initial steady state which is perturbed by suddenly changing the surface velocity $U_{0}$ to $U$ or the angular velocity of the fluid $\Omega_{0}$ to $\Omega$. This impulsive change causes unsteadiness in the flow field. The parabolic partial differential equations governing the flow and heat transfer have been solved by using an implicit finite-difference scheme in combination with the quasilinearization technique $[12,13]$. The computations have been carried out from time $t=0$ to the final steady state. The results have been compared with those of Wang [10]. The present results will be useful in controlling the growth of the secondary flow caused by the rotation of the fluid by applying magnetic field.

\section{Formulation and analysis}

Let us consider the unsteady motion of a viscous incompressible electrically conducting fluid induced by suddenly moving the surface with a constant velocity $U$ from its initial steady-state value $U_{0}$ or by impulsively changing the angular velocity of the fluid to $\Omega$ from its 
initial value $\Omega_{0}$. These sudden changes introduce unsteadiness in the flow field. The surface coincides with plane $z=0$ and it is moved with a constant velocity $U$ in $x$ direction. The fluid is rotating with a constant angular velocity $\Omega$ about the $z$-axis. The moving surface is also rotating with the fluid. The flow is three-dimensional due to the presence of the Coriolis force. The surface temperature and the fluid temperature at the edge of the boundary layer are constants. Fig. 1 shows the coordinate system, where $x, y$ and $z$ are the Cartesian coordinates and $u, v$ and $w$ are the velocity components along $x, y$ and $z$ directions, respectively. Since the flow is induced by the movement of the surface in $x$ direction only, the velocity components $u, v$ and $w$ and the temperature $T$ depend on $x, z$ and $t$. The magnetic field $B$ is applied in $z$ direction. It is assumed that the magnetic Reynolds number $R e_{\mathrm{m}}=\mu_{0} \sigma V L \ll 1$, where $\mu_{0}$ and $\sigma$ are magnetic permeability and electrical conductivity, respectively and $V$ and $L$ are the characteristic velocity and length, respectively. Under this condition it is possible to neglect the induced magnetic field in comparison to the applied magnetic field. The external electric field as well as the induced electric field have also been neglected $[14,15]$. The viscous dissipation and Ohmic heating have been neglected in the energy equation. Under the above assumptions, the unsteady boundary layer equations based on the conservation of mass, momentum and energy in a rotating frame of reference can be expressed as $[10,11,16]$.

$$
\begin{aligned}
& u_{x}+w_{z}=0, \\
& u_{t}+u u_{x}+w u_{z}-2 \Omega v=v u_{z z}-\rho^{-1} \sigma B^{2} u, \\
& v_{t}+u v_{x}+w v_{z}+2 \Omega u=v v_{z z}-\rho^{-1} \sigma B^{2} v, \\
& T_{t}+u T_{x}+w T_{z}=\alpha T_{z z} .
\end{aligned}
$$

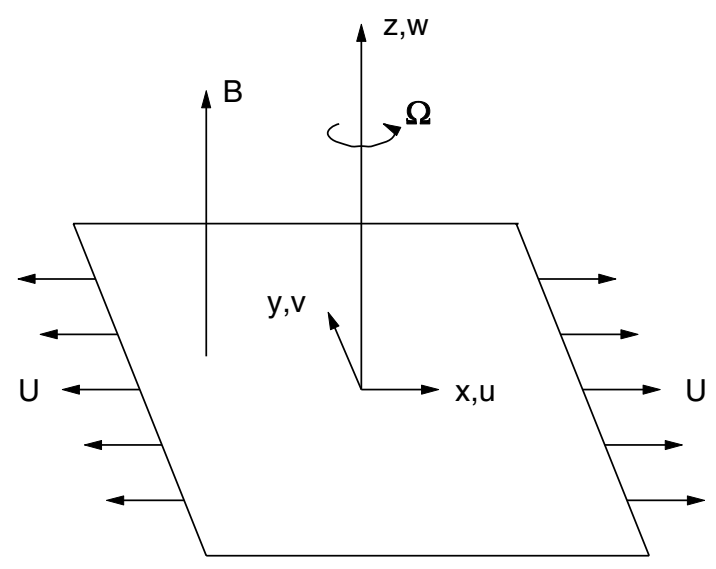

Fig. 1. Physical model and coordinate system.
The initial conditions are

$u(x, z, 0)=u_{i}(x, z), \quad v(x, z, 0)=v_{i}(x, z)$,
$w(x, z, 0)=w_{i}(x, z), \quad T(x, z, 0)=T_{i}(x, z)$

and the boundary conditions for $t>0$ are

$u(x, 0, t)=U, \quad v(x, 0, t)=w(x, 0, t)=0$,

$T(x, 0, t)=T_{\mathrm{w}}, \quad u(x, \infty, t)=v(x, \infty, t)=0$,

$T(x, \infty, t)=T_{\infty}$.

It is convenient to convert Eqs. (1)-(4) from $(x, z, t)$ system to $\left(\eta, t^{*}\right)$ system by using the following transformations

$\eta=(a / v)^{1 / 2} z, \quad t^{*}=a t, \quad u=a x f^{\prime}\left(\eta, t^{*}\right)$,

$v=\operatorname{axs}\left(\eta, t^{*}\right), \quad w=-(a v)^{1 / 2} f\left(\eta, t^{*}\right)$,

$T-T_{\infty}=\left(T_{\infty}-T_{\mathrm{w}}\right) \theta\left(\eta, t^{*}\right), \lambda=\Omega_{0} / a$,

$U=U_{0}\left(1+\epsilon_{1}\right), \quad U_{0}=a x, \quad \Omega=\Omega_{0}\left(1+\epsilon_{2}\right)$,

$\operatorname{Pr}=v / \alpha, \quad M=\sigma B^{2} / \rho a$,

to them and we find that (1) is identically satisfied and (2)-(4) reduce to

$f^{\prime \prime \prime}+f f^{\prime \prime}-f^{\prime 2}+2 \lambda s-M f^{\prime}-\partial f^{\prime} / \partial t^{*}=0$,

$s^{\prime \prime}+f s^{\prime}-f^{\prime} s-2 \lambda f^{\prime}-M s-\partial s / \partial t^{*}=0$,

$P r^{-1} \theta^{\prime \prime}+f \theta^{\prime}-\partial \theta / \partial t^{*}=0$,

with boundary conditions

$f\left(0, t^{*}\right)=f^{\prime}\left(0, t^{*}\right)-\left(1+\epsilon_{1}\right)=s\left(0, t^{*}\right)=\theta\left(0, t^{*}\right)=0$,

$f^{\prime}\left(\infty, t^{*}\right)=s\left(\infty, t^{*}\right)=\theta\left(\infty, t^{*}\right)-1=0$.

The initial conditions are given by the steady-state equations along with boundary conditions obtained from (8)-(11) by putting $t^{*}=\epsilon_{1}=\partial f^{\prime} / \partial t^{*}=\partial s / \partial t^{*}=\partial \theta /$ $\partial t^{*}=0$. The steady state equations are given by

$f^{\prime \prime \prime}+f f^{\prime \prime}-f^{\prime 2}+2 \lambda s-M f^{\prime}=0$,

$s^{\prime \prime}+f s^{\prime}-f^{\prime} s-2 \lambda f^{\prime}-M s=0$,

$\operatorname{Pr}^{-1} \theta^{\prime \prime}+f \theta^{\prime}=0$,

with boundary conditions

$f=f^{\prime}-1=s=\theta=0$ at $\eta=0$,

$f^{\prime}=s=\theta-1=0$ as $\eta \rightarrow \infty$.

When the unsteadiness in the flow field is caused by impulsively changing the angular velocity of the fluid from $\Omega_{0}$ to $\Omega\left(=\Omega_{0}\left(1+\epsilon_{2}\right)\right)$, the equations governing this case are the same as (8)-(11) except that $\lambda$ in (8) and (9) is replaced by $\lambda\left(1+\epsilon_{2}\right)$ and $\epsilon_{1}=0$ in (11). The initial conditions are given by (12)-(15).

It may be noted that the steady-state equations (12)-(15) for $M=0$ (in the absence of the magnetic field) are identical to those of Wang [10]. 
The local skin friction coefficients for the primary and secondary flows $\left(C_{\mathrm{f} x}, C_{\mathrm{fz}}\right)$ and the local Nusselt number $\left(N u_{x}\right)$ are given by

$$
\begin{aligned}
& C_{f x}=-\mu(\partial u / \partial z)_{z=0} / \rho U_{0}^{2}=-R e_{x}^{-1 / 2} f^{\prime \prime}\left(0, t^{*}\right), \\
& C_{f z}=-\mu(\partial v / \partial z)_{z=0} / \rho U_{0}^{2}=-\operatorname{Re}_{x}^{-1 / 2} s^{\prime}\left(0, t^{*}\right), \\
& N u_{x}=x(\partial T / \partial z)_{z=0} /\left(T_{\infty}-T_{\mathrm{w}}\right)=\operatorname{Re}_{x}^{1 / 2} \theta^{\prime}\left(0, t^{*}\right) .
\end{aligned}
$$

\section{Analytical solution}

It is possible to obtain analytical solutions for the final steady-state equations (8) and (10) for the primary flow with $\lambda=0 \quad(s=0), \quad t^{*} \rightarrow \infty, \quad \partial f^{\prime} / \partial t^{*}=\partial \theta / \partial t^{*}=0$ under relevant boundary conditions given in (11). The solution of (8) satisfying the boundary conditions can be expressed as

$f(\eta)=\left(1+\epsilon_{1}\right) \beta^{-1}\left(1-\mathrm{e}^{-\beta \eta}\right)$,
$\beta=\left(1+\epsilon_{1}+M\right)^{1 / 2}>0$,

Using (17) in (10), we get

$\theta^{\prime \prime}+\operatorname{Pr}\left(1+\epsilon_{1}\right) \beta^{-1}\left(1-\mathrm{e}^{-\beta \eta}\right) \theta^{\prime}=0$,

with boundary conditions

$\theta(0)=0, \quad \theta(\infty)=1$. by

We now change the independent variable $\eta$ to $\xi$ given

$\xi=-A \mathrm{e}^{-\beta \eta}, \quad A=\operatorname{Pr}\left(1+\epsilon_{1}\right) \beta^{-2}>0$.

From (18) and (19), we obtain

$\xi \frac{\mathrm{d}^{2} \theta}{\mathrm{d} \xi^{2}}+(1-A-\xi) \frac{\mathrm{d} \theta}{\mathrm{d} \xi}=0$,

with boundary conditions

$\theta(-A)=0, \quad \theta(0)=1$.

The solution of (20) is given in terms of Kummer's function $H$ [17]

$\theta(\xi)=1-(-\xi / A)^{A}[H(A, A+1, \xi) / H(A, A+1,-A)]$.
In terms of $\eta$, (21) can be expressed as

$\theta(\eta)=1-\mathrm{e}^{-A \beta \eta}\left[H\left(A, A+1,-A \mathrm{e}^{-\beta \eta}\right) / H(A, A+1,-A)\right]$,

where

$H(a, b, z)=1+\sum_{j=1}^{\infty} \frac{(a)_{j} z^{j}}{(b)_{j} j !}$,

$(a)_{j}=a(a+1)(a+2) \ldots(a+j-1)$,

$(b)_{j}=b(b+1)(b+2) \ldots(b+j-1)$.

For $\epsilon_{1}=m=0, \operatorname{Pr}=\beta=1$, (22) reduces to a simple form

$\theta(\eta)=1-e(e-1)^{-1}\left[1-\exp \left(-\mathrm{e}^{-\eta}\right)\right]$.

The above results also hold good for $t^{*}=0$, but one has to put $\epsilon_{1}=0$.

\section{Results and discussion}

Eqs. (8)-(10) under boundary conditions (11) and initial conditions (12)-(15) have been solved by using an implicit finite-difference method in combination with the quasilinearization technique $[12,13]$. The three-point central-difference formula has been used in $\eta$ direction and the two-point backward difference formula in $t^{*}$ direction. The step sizes used here are as follows: $\Delta t^{*}=0.0005 \quad$ for $0 \leqslant t^{*} \leqslant 0.05, \quad \Delta t^{*}=0.005$ for $0.05<t^{*} \leqslant 0.1, \Delta t^{*}=0.01$ for $0.1<t^{*} \leqslant 0.5, \Delta t^{*}=0.05$ for $t^{*}>0.5, \Delta \eta=0.05$ and $\eta_{\infty}=10$. These step sizes have been selected after carrying out the sensitivity analysis on the solutions.

In order to assess the accuracy of our method, we have compared our steady-state results $\left(-f^{\prime \prime}(0),-s^{\prime}(0), \theta^{\prime}(0)\right)$ for $M=0$ with those of Wang [10]. The results are found to be in excellent agreement. The comparison is presented in Table 1. The results for the case where the impulsive motion is imported to the surface velocity are given in Figs. 2-8 and for the case where the impulsive motion is given to the angular velocity of the fluid in Figs. 9-14.

The variation of the surface shear stress for the primary flow $\left(-f^{\prime \prime}\left(0, t^{*}\right)\right)$ with time $t^{*}\left(0<t^{*} \leqslant 1.0\right)$ for

Table 1

Comparison of surface shear stresses and heat transfer results $\left(-f^{\prime \prime}(0),-s^{\prime}(0), \theta^{\prime}(0)\right)$ with those of Wang $[10]$ for $t^{*}=\epsilon_{1}=\epsilon_{2}=M=0$

\begin{tabular}{llllll}
\hline$\lambda$ & $-f^{\prime \prime}(0)$ & $-s^{\prime}(0)$ & $\theta^{\prime}(0)$ & & \\
\cline { 3 - 6 } & & & $\operatorname{Pr}=0.7$ & $\operatorname{Pr}=2.0$ & \\
\hline 0.0 & $0.9999(1.0000)^{\mathrm{a}}$ & $0.0000(0.0000)$ & $0.4560(0.4550)$ & $0.9117(0.9110)$ & $1.8977(1.8940)$ \\
0.5 & $1.1382(1.1384)$ & $0.5124(0.5128)$ & $0.3903(0.3900)$ & $0.8530(0.8530)$ & $1.8561(1.8500)$ \\
1.0 & $1.3251(1.3250)$ & $0.8362(0.8371)$ & $0.3253(0.3210)$ & $0.7704(0.7700)$ & $1.7921(1.7880)$ \\
2.0 & $1.6535(1.6523)$ & $1.2851(1.2873)$ & $0.2549(0.2420)$ & $0.6382(0.6380)$ & $1.6684(1.6640)$ \\
\hline
\end{tabular}

${ }^{a}$ Results obtained by Wang [10]. 


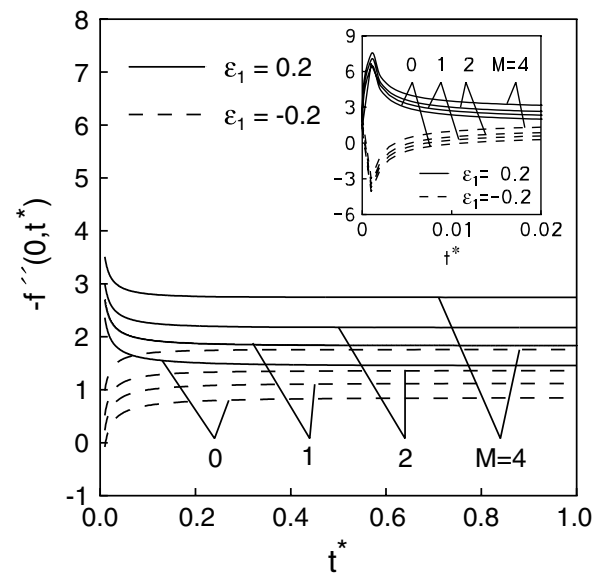

Fig. 2. Variation of the surface shear stress for the primary flow, $-f^{\prime \prime}\left(0, t^{*}\right)$, with time $t^{*}$ for $\epsilon_{1}= \pm 0.2, \epsilon_{2}=0,0 \leqslant M \leqslant 4$, $\lambda=0.5$.

$\epsilon_{1}= \pm 0.2, M=0,1,2,4, \epsilon_{2}=0, \lambda=0.5$ is given in Fig. 2. The inset shows the variation of $\left(-f^{\prime \prime}\left(0, t^{*}\right)\right)$ with $t^{*}$ in a small time interval $0 \leqslant t^{*} \leqslant 0.02$. It is found that for $t^{*}>1$, the surface shear stresses $\left(-f^{\prime \prime}\left(0, t^{*}\right),-s^{\prime}\left(0, t^{*}\right)\right)$ change little with time $t^{*}$. Hence the results are shown in the time interval $0 \leqslant t^{*} \leqslant 1$. Since the heat transfer $\left(\theta^{\prime}\left(0, t^{*}\right)\right)$ takes more time to reach the steady state, it is shown in the range $0 \leqslant t^{*} \leqslant 5$. For $\epsilon_{1}=-0.2$ (when the surface velocity is suddenly reduced at $t^{*}>0$ ), in a small time interval $0<t^{*}<0.01,-f^{\prime \prime}\left(0, t^{*}\right)$ changes from a positive to a negative value due to the change in the sign of the velocity gradient at the wall as explained earlier. However, the vanishing of the shear stress does not imply separation, because we are considering unsteady flow over a moving surface [18]. On the other hand, for $\epsilon_{1}=0.2$ (when the wall velocity is suddenly increased), there is no change in the sign of the surface shear stress, i.e., $-f^{\prime \prime}\left(0, t^{*}\right)>0$ for all times for the reasons explained earlier. Since the surface velocity is impulsively changed at $t^{*}>0$, the surface shear stress $f^{\prime \prime}\left(0, t^{*}\right)$ changes significantly in a small time interval after the start of the impulsive motion. The surface shear stress for $\epsilon_{1}=0.2$ is more than that of $\epsilon_{1}=-0.2$, because the boundary layer for $\epsilon_{1}=0.2$ is thinner than that for $\epsilon_{1}=-0.2$. This reduction is due to the fluid acceleration induced by the increase in the surface velocity. For a fixed $t^{*}$, the surface shear stress $f^{\prime \prime}\left(0, t^{*}\right)$ increase with the magnetic parameter $M$ due to the Lorentz magnetic force.

The variation of the surface shear stresses for the secondary flow $\left(-s^{\prime}\left(0, t^{*}\right)\right)$ and the surface heat transfer $\left(\theta^{\prime}\left(0, t^{*}\right)\right)$ with time $t^{*}$ for $\epsilon_{1}= \pm 0.2, \epsilon_{2}=0,0 \leqslant M \leqslant 4$, $\lambda=0.5$ is presented in Figs. 3 and 4 , respectively. The surface shear stress for the secondary flow and the surface heat transfer change monotonically with time $t^{*}$

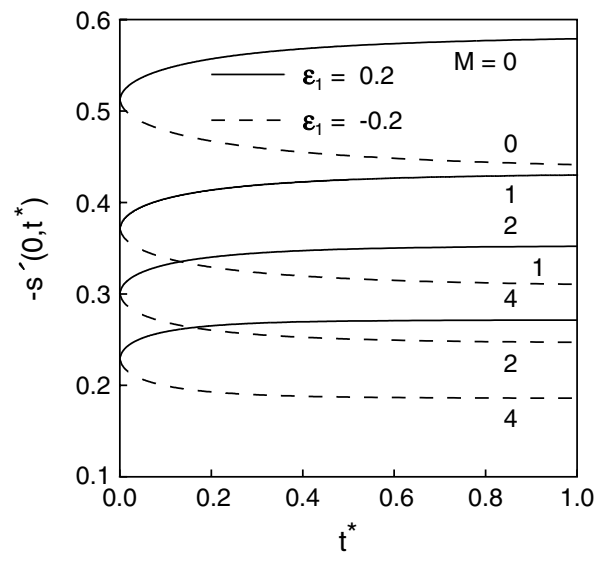

Fig. 3. Variation of the surface shear stress for the secondary flow, $-s^{\prime}\left(0, t^{*}\right)$, with time $t^{*}$ for $\epsilon_{1}= \pm 0.2, \epsilon_{2}=0,0 \leqslant M \leqslant 4$, $\lambda=0.5$.

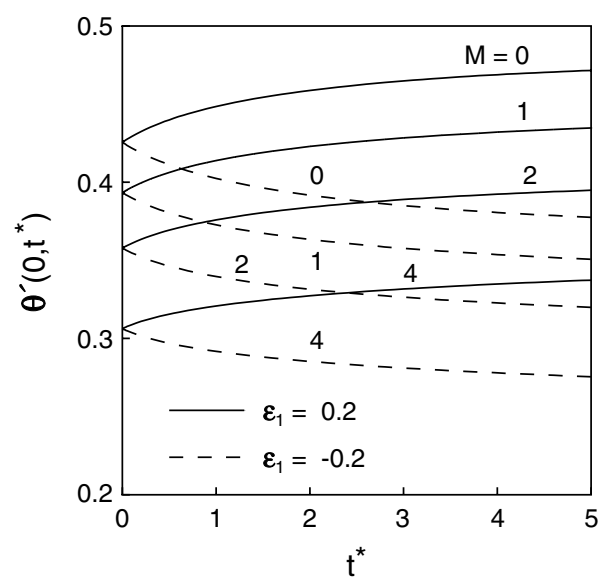

Fig. 4. Variation of the surface heat transfer, $\theta^{\prime}\left(0, t^{*}\right)$, with time $t^{*}$ for $\epsilon_{1}= \pm 0.2, \epsilon_{2}=0,0 \leqslant M \leqslant 4, \lambda=0.5, \operatorname{Pr}=0.78$.

and the steady state is reached rather quickly. Unlike the shear stress for the primary flow, the surface shear stress for the secondary flow and the surface heat transfer are comparatively less affected by the impulsive change in the surface velocity, because its effect on them is indirect. Since the magnetic field has stabilizing effect on the flow field, it retards the growth of the secondary flow. Consequently, the surface shear stress for the secondary flow $\left(-s^{\prime}\left(0, t^{*}\right)\right)$ decreases with increasing $M$. The surface heat transfer $\left(\theta^{\prime}\left(0, t^{*}\right)\right)$ also decreases with increasing $M$. The reduction in the heat transfer is due to the reduction in the axial velocity $f$. The transition from the unsteady to the final steady flow takes place continuously without any singularity. The surface heat transfer takes more time to reach the steady state than the surface shear stresses. 
The effect of the rotation parameter $\lambda$ on the surface shear stresses for the primary and secondary flows $\left(-f^{\prime \prime}\left(0, t^{*}\right),-s^{\prime}\left(0, t^{*}\right)\right)$ and the surface heat transfer $\left(\theta^{\prime}\left(0, t^{*}\right)\right)$ for $\epsilon_{1}= \pm 0.2, \epsilon_{2}=0, M=1, \operatorname{Pr}=0.78$ is displayed in Figs. 5-7. The surface shear stresses increase with the rotation parameter $\lambda$, but the heat transfer decreases. Since the increase in the angular velocity of the fluid accelerates the fluid, the surface shear stresses increase. However, the heat transfer on the surface decreases due to the reduction in the axial velocity $f$.

The effect of the Prandtl number $\operatorname{Pr}(\operatorname{Pr}=0.027$ for mercury and $P r=0.78$ for lithium-ammonia) on the surface heat transfer $\left(\theta^{\prime}\left(0, t^{*}\right)\right)$ for $\epsilon_{1}= \pm 0.2, \epsilon_{2}=0$,

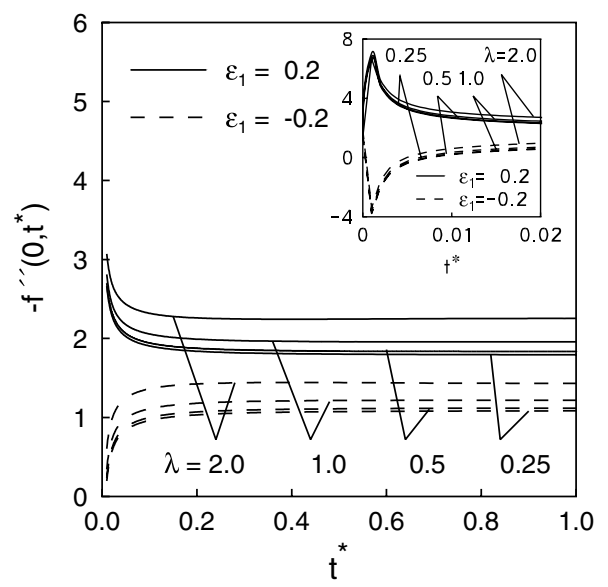

Fig. 5. Variation of the surface shear stress for the primary flow, $-f^{\prime \prime}\left(0, t^{*}\right)$, with time $t^{*}$ for $\epsilon_{1}= \pm 0.2, \epsilon_{2}=0, \quad M=1$, $0.25 \leqslant \lambda \leqslant 2.0$.

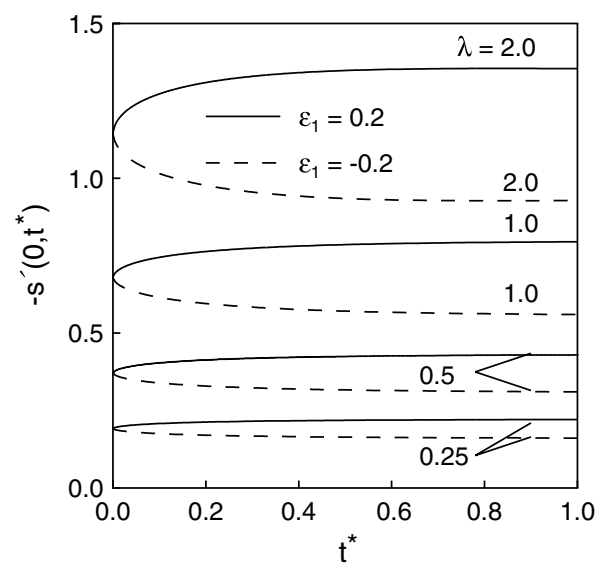

Fig. 6. Variation of the surface shear stress for the secondary flow, $-s^{\prime}\left(0, t^{*}\right)$, with time $t^{*}$ for $\epsilon_{1}= \pm 0.2, \epsilon_{2}=0, M=1$, $0.25 \leqslant \lambda \leqslant 2.0$.

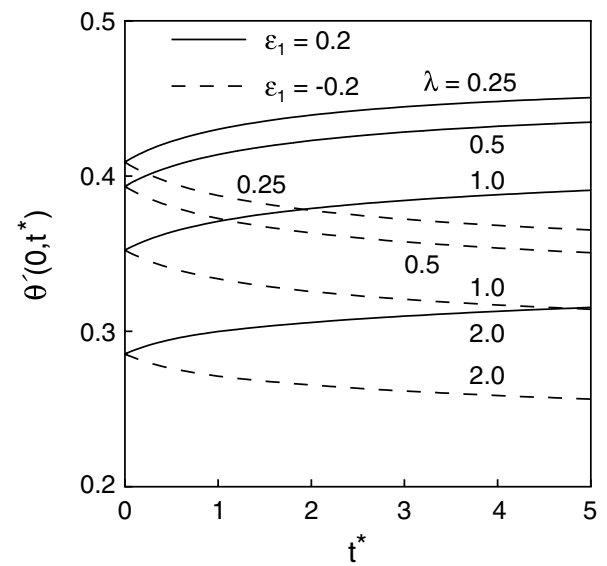

Fig. 7. Variation of the surface heat transfer, $\theta^{\prime}\left(0, t^{*}\right)$, with time $t^{*}$, for $\epsilon_{1}= \pm 0.2, \epsilon_{2}=0, M=1,0.25 \leqslant \lambda \leqslant 2.0$.

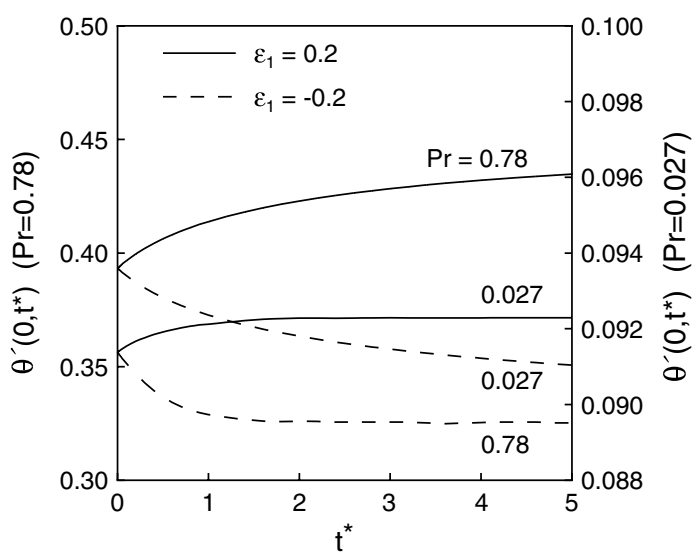

Fig. 8. Effect of the Prandtl number, $P r$, on the surface heat transfer, $\theta^{\prime}\left(0, t^{*}\right)$, for $\epsilon_{1}= \pm 0.2, \epsilon_{2}=0, M=1, \lambda=0.5$.

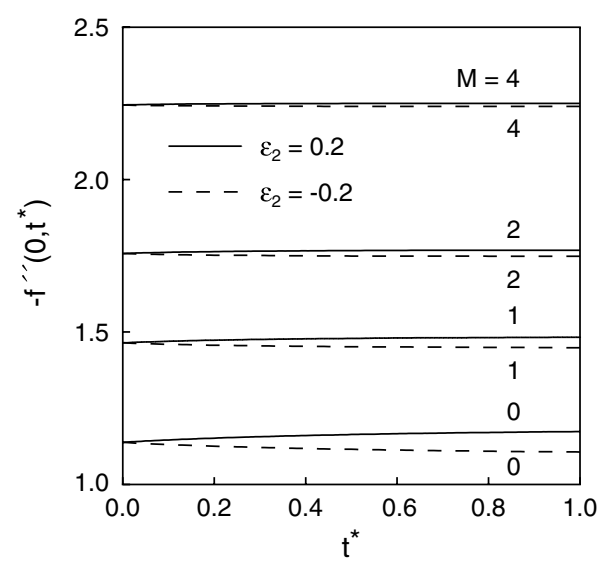

Fig. 9. Variation of the surface shear stress for the primary flow, $-f^{\prime \prime}\left(0, t^{*}\right)$, with time $t^{*}$ for $\epsilon_{2}= \pm 0.2, \epsilon_{1}=0,0 \leqslant M \leqslant 4$, $\lambda=0.5$. 
$M=1, \lambda=0.5$ is presented in Fig. 8. Since increase in $\mathrm{Pr}$ results in thinner thermal boundary layer, the surface heat transfer increases with $\mathrm{Pr}$ for all $t^{*}$. For a fixed $P r$, it increases with $t^{*}$ when the surface velocity is increased $\left(\epsilon_{1}>0\right)$, but it decreases with increasing $t^{*}$ for $\epsilon_{1}<0$ (when the surface velocity is suddenly reduced). The reason for this behaviour is already explained.

The variation of the surface shear stress for the primary and secondary flows $\left(-f^{\prime \prime}\left(0, t^{*}\right),-s^{\prime}\left(0, t^{*}\right)\right)$ and the surface heat transfer $\left(\theta^{\prime}\left(0, t^{*}\right)\right)$ with time $t^{*}$ for $\epsilon_{2}= \pm 0.2$ (when the impulsive motion is applied to the angular velocity of the fluid), $0 \leqslant M \leqslant 4, \epsilon_{1}=0$, $\lambda=0.5, P r=0.78$ is shown in Figs. 9-11. Since magnetic field has a stabilizing effect on the flow field, it enhances the surface shear stress for the primary flow $\left(-f^{\prime \prime}\left(0, t^{*}\right)\right)$,

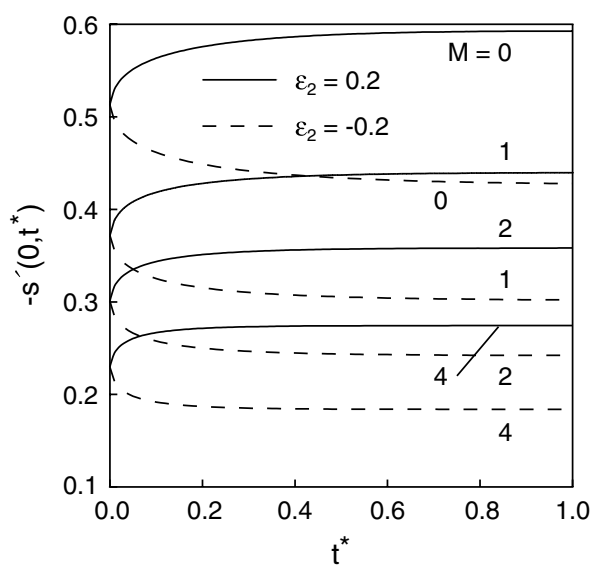

Fig. 10. Variation of the surface shear stress for the secondary flow, $-s^{\prime}\left(0, t^{*}\right)$, with time $t^{*}$ for $\epsilon_{2}= \pm 0.2, \epsilon_{1}=0,0 \leqslant M \leqslant 4$, $\lambda=0.5$.

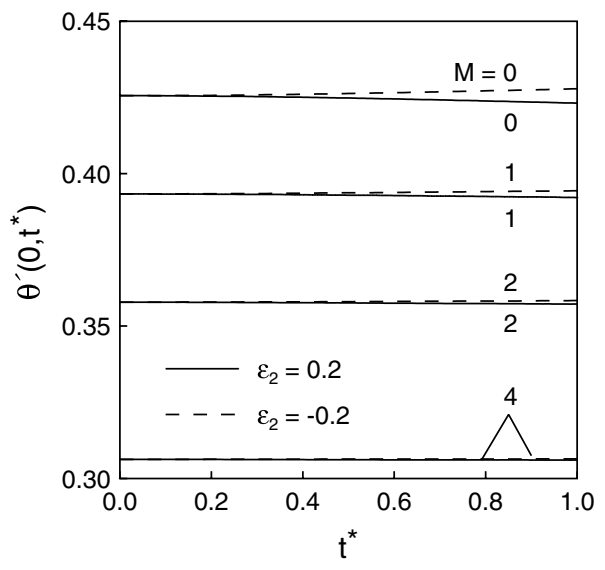

Fig. 11. Variation of the surface heat transfer, $\theta^{\prime}\left(0, t^{*}\right)$, with time $t^{*}$ for $\epsilon_{2}= \pm 0.2, \epsilon_{1}=0,0 \leqslant M \leqslant 4, \lambda=0.5, \operatorname{Pr}=0.78$. but reduces the surface shear stress for the secondary flow $\left(-s^{\prime}\left(0, t^{*}\right)\right)$. Further, it also reduces the surface heat transfer $\left(\theta^{\prime}\left(0, t^{*}\right)\right)$ due to the reduction in the axial velocity $f$. Since impulsive motion is given to the rotating fluid, the effect of time variation is pronounced only on the surface shear stress for the secondary flow $\left(-s^{\prime}\left(0, t^{*}\right)\right)$ in a small time interval $0 \leqslant t^{*}<0.2$. The steady state is reached quickly.

Figs. 12-14 present the effect of the rotation parameter $\lambda$ on the surface shear stresses for the primary and secondary flows $\left(-f^{\prime \prime}\left(0, t^{*}\right),-s^{\prime}\left(0, t^{*}\right)\right)$ and the surface heat transfer $\left(\theta^{\prime}\left(0, t^{*}\right)\right)$ for $\epsilon_{2}= \pm 0.2, \epsilon_{1}=0, M=1$, $\operatorname{Pr}=0.78$. Since the rotation of the fluid $(\lambda)$ gives rise to the secondary flow, the surface shear stresses for the primary and secondary flows increase with $\lambda$. Since

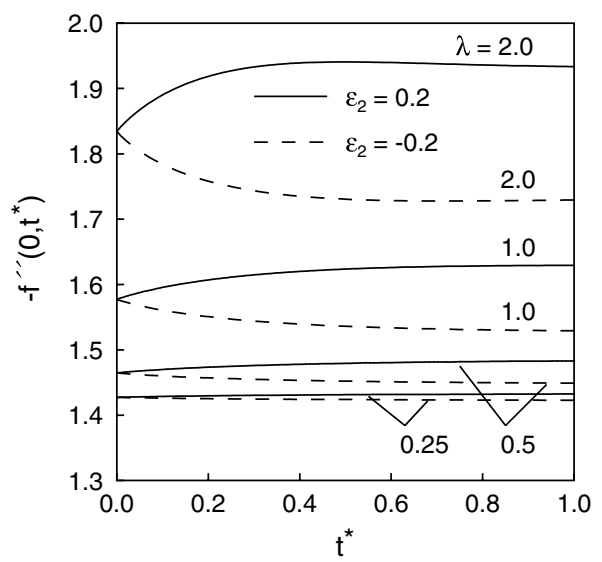

Fig. 12. Effect of the rotation parameter, $\lambda$, on the surface shear stress for the primary flow, $-f^{\prime}\left(0, t^{*}\right)$, for $\epsilon_{2}= \pm 0.2, \epsilon_{1}=0$, $M=1$.

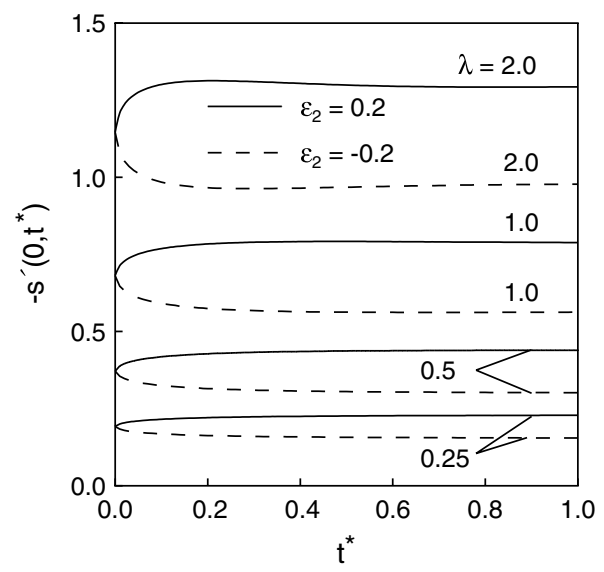

Fig. 13. Effect of the rotation parameter, $\lambda$, on the surface shear stress for the secondary flow, $-s^{\prime}\left(0, t^{*}\right)$, for $\epsilon_{2}= \pm 0.2, \epsilon_{1}=0$, $M=1$. 


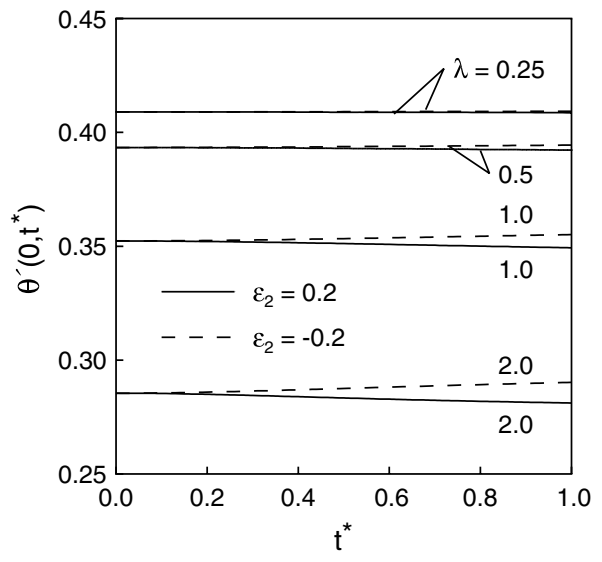

Fig. 14. Effect of the rotation parameter, $\lambda$, on the surface heat transfer, $\theta^{\prime}\left(0, t^{*}\right)$, for $\epsilon_{2}= \pm 0.2, \epsilon_{2}=0, M=1, \operatorname{Pr}=0.78$.

the axial velocity $f$ decreases with increasing $\lambda$ due to the reduction of the boundary layer thickness, the surface heat transfer decreases with increasing $\lambda$.

\section{Conclusions}

The magnetic field and the rotation parameter exert a strong influence on the surface shear stresses for the primary and secondary flows and the surface heat transfer. When the surface velocity is suddenly reduced, the surface shear stress for the primary flow vanishes in a small time interval after the start of the impulsive motion. However, it does not imply flow separation, because we are considering unsteady flow over a moving surface. Significant changes in the flow and heat transfer take place only in a small time interval after the start of the impulsive motion and the final steady state is reached quickly, i.e. spin-up or spin-down time is rather small. In general, the surface heat transfer takes longer time to reach the steady state than the surface shear stresses.

\section{Acknowledgement}

One of the authors (MK) is thankful to the University Grants Commission, India, for the financial support under the Research Scientist Scheme.

\section{References}

[1] B.C. Sakiadis, Boundary layer behaviour on continuous solid surface II. The boundary layer on a continuous flat surface, Am. Inst. Chem. Eng. J. 7 (1961) 221-225.

[2] F.K. Tsou, E.M. Sparrow, R.J. Goldstein, Flow and heat transfer in the boundary layer on a continuous moving surface, Int. J. Heat Mass Transfer 10 (1967) 219-235.

[3] L.J. Crane, Flow past a stretching plate, Z. Angew. Math. Phys. 21 (1970) 445-447.

[4] P. Carragher, L.J. Crane, Heat transfer on a continuous stretching sheet, Z. Angew. Math. Mech. 82 (1982) 964 965.

[5] A. Chakrabarti, A.S. Gupta, Hydromagnetic flow and heat transfer over a stretching sheet, Quart. Appl. Math. 37 (1977) 73-78.

[6] H.I. Andersson, MHD flow of a viscous fluid past a stretching surface, Acta Mech. 95 (1992) 227-230.

[7] K. Vajravelu, A. Hadjinicolaou, Convective heat transfer in an electrically conducting fluid at a stretching surface with uniform free stream, Int. J. Eng. Sci. 35 (1997) 12371244.

[8] I. Pop, T.Y. Na, A note on MHD flow over a stretching permeable surface, Mech. Res. Commun. 25 (1998) 263269.

[9] M. Kumari, G. Nath, Flow and heat transfer in a stagnation-point flow over a stretching sheet with a magnetic field, Mech. Res. Commun. 26 (1999) 469-478.

[10] C.Y. Wang, Stretching a surface in a rotating fluid, Z. Angew. Math. Phys. 39 (1988) 177-185.

[11] H.S. Takhar, G. Nath, Unsteady flow over a stretching surface with a magnetic field in a rotating fluid, Z. Angew. Math. Phys. 49 (1998) 989-1001.

[12] J.R. Radbill, G.A. McCue, Quasilinearization in Nonlinear Problems in Fluid and Orbital Mechanics, Elsevier, New York, 1970.

[13] K. Inouye, A. Tate, Finite-difference version quasilinearization applied to boundary layer equations, AIAA J. 12 (1974) 558-560.

[14] J.L. Neuringer, W. McIlroy, Incompressible two-dimensional stagnation-point flow of an electrically conducting viscous fluid in the presence of a magnetic field, $J$. Aeronaut. Sci. 25 (1958) 194-198.

[15] V.J. Rossow, Magnetohydrodynamic analysis of heat transfer near a stagnation point, J. Aeronaut Sci. 25 (1958) 234-235.

[16] A.C. Eringen, G.A. Maugin, Electrodynamics in Continua, vol.2, Springer, Berlin, 1990.

[17] M. Abramowitz, I.A. Stegun, Handbook of Mathematical Functions, vol. 55, Providence, National Bureau of Standards, American Mathematical Society, 1972.

[18] F.T. Smith, Steady and unsteady boundary-layer separation, Ann. Rev. Fluid Mech. 18 (1986) 197-220. 